Pacific Journal of Mathematics

PRIMARY RINGS AND DOUBLE CENTRALIZERS 


\title{
PRIMARY RINGS AND DOUBLE CENTRALIZERS
}

\author{
Kent R. Fuller
}

\begin{abstract}
This note is devoted to proving the theorem that every right quasi-projective module over a semi-primary ring $R$ has the double centralizer property if and only if $R$ is a direct sum of primary rings, and to discussing some of its consequences. In particular, this theorem places a strong necessary condition on a large class of the balanced rings of Camillo which are both a specialization of Thrall's $Q F-1$ rings and a generalization of the uniserial rings of Köthe.
\end{abstract}

Let $R$ be an associative ring with identity. An $R$-module $M$ is quasi-projective [19] (quasi-injective [10]) in case $\operatorname{Hom}_{R}(M, \quad)\left(\mathrm{Hom}_{R}\right.$ $(, M)$ ) preserves the exactness of all short exact sequences with middle term $M$. If the natural ring homomorphism of $R$ into the double centralizer, $\operatorname{Hom}_{C}(M, M)$, where $C=\operatorname{Hom}_{R}(M, M)$, is onto, the $R$-module $M$ is said to have the double centralizer property.

Let $N$ denote the Jacobson radical of $R$. Then $R$ is semi-primary in case $N$ is nilpotent and $R / N$ is semi-simple. If, in addition, $R / N$ is a simple ring, $R$ is said to be primary. A semi-primary ring $R$ is a direct sum of primary rings if and only if for each pair of primitive idempotents $e$ and $f$ in $R f N e \neq 0$ implies $f R \cong e R$. In other words, direct sums of primary rings are just those semi-primary rings in which the composition factors in each primitive one-sided ideal are pairwise isomorphic. It follows that if $N$ is nilpotent and $R / N^{2}$ is a direct sum of primary rings, then so is $R$.

We shall need the following notation. The socle, $S(M)$, of an $R$ module $M$ is its largest semi-simple submodule. The top, $T(M)$, of $M$ is the largest semi-simple factor module of $M$. The former is equal to the annihilator in $M$ of $N$, the latter is $M / N M$ (or $M / M N$ ). In particular, if $e$ is a primitive idempotent in $R$ then $T(R e)$ is the unique simple factor module of $R e$.

With the above, we are ready to prove the main result.

THEOREM. Every right (equivalently, left) quasi-projective module over a semi-primary ring $R$ has the double centralizer property if and only if $R$ is a direct sum of primary rings.

Proof. $(\Longrightarrow)$ Let $R$ be semi-primary. In view of the above comments and the fact that quasi-projective modules over factor rings of $R$ are quasi-projective as $R$-modules, we may assume that $N^{2}=0$. Suppose that, for some primitive idempotents $e$ and $f$ in $R$, 
$f N e \neq 0$ and $f R \neq e R$. We prove this implication by constructing a factor ring $\hat{R}$ of $R$ over which some faithful projective right module does not have the double centralizer property. To this end, let $A=$ $\{r \in R \mid f R r=0\}$, the right annihilator in $R$ of $f R$. Write $\bar{R}=R / A$, $\bar{N}=(N+A) / A, \bar{e}=e+A$ and $\bar{f}=f+A$. Here $\bar{N}$ is the radical of $\bar{R}$ and, because $f R \cap A=0, \bar{e}$ and $\bar{f}$ are primitive idempotents in $\bar{R}$ with $\bar{f} \bar{N} \bar{e} \neq 0$. Now repeat the process on the left to construct from $\bar{R}$ and $\bar{B}=\{\bar{r} \in \bar{R} \mid \bar{r} \bar{R} \bar{e}=0\}$ a factor ring $\hat{R}$ possessing radical $\hat{N}$ and primitive idempotents $\hat{f}$ and $\hat{e}$ with $\hat{f} \hat{N} \hat{e} \neq 0$. Since the modules $\bar{f} \bar{R}_{\bar{R}}$ and ${ }_{\hat{R}} \hat{R} \hat{e}$ are faithful it follows, as in the discussion preceding [8, Th. 3], that every minimal left ideal in $\bar{R}$ is isomorphic to $T(\bar{R} \bar{f})$ and every minimal right ideal in $\hat{R}$ is isomorphic to $T(\hat{e} \hat{R})$. Thus, noting that $\bar{N} \cong S\left(_{\bar{R}} \bar{R}\right)$ and $\hat{N} \subseteq S\left(\hat{R}_{\hat{R}}\right)$, we glean the additional information that $\hat{e} \hat{N}=0$ and $\hat{N} \hat{f}=0$. According to the implication $(\mathrm{b}) \Rightarrow(\mathrm{d})$ of [8, Th. 4] (whose proof is valid for semiprimary rings), the present proof will be complete once we show that $T(\hat{R} \hat{e})$ is not isomorphic to a minimal left ideal in $\hat{R}$ and that $\operatorname{Ext}_{\hat{R}}^{1}(T(\hat{R} \hat{e}), \hat{R}) \neq 0$. Because $\hat{N}^{2}=0$ we have $S(\hat{R} \hat{R})=\hat{N}+\hat{T}$ where $\hat{T}$ is the sum of the simple primitive left ideals in $\hat{R}$. But $\hat{e} \hat{N}=0$ and $\hat{N} \hat{e} \neq 0$ so $T(\hat{R} \hat{e})$ cannot be embedded in either summand. On the other hand, $\hat{N} \hat{f}=0$ implies that $T(\hat{R} \hat{f})$ is a direct summand of ${ }_{\hat{R}} \hat{R}$, so an essential extension ${ }_{\hat{R}} M$ of $T(\hat{R} \hat{f})$ with $M / T(\hat{R} \hat{f}) \cong T(\hat{R} \hat{e})$ will show that $\operatorname{Ext}_{R}^{1}(T(\hat{R} \hat{e}), R) \neq 0$. To obtain such an extension, let $E=E(T(\hat{R} \hat{f}))$, the injective hull [4] of $T(\hat{R} \hat{f})$ over $\hat{R}$. Then since $\hat{f} \hat{R} \hat{e} \neq 0$ and $\hat{e} \hat{R} \hat{f}=0$ we have, by [7, Lemma (1.1), (c)], that $\hat{e} E / T(\hat{R} \hat{f}) \neq 0$. This and the fact that $E / T(\hat{R} \hat{f})$ is semi-simple yield an $\hat{R}$-module $M$ with $T(\hat{R} \hat{f}) \leqq M \leqq E$ and $M / T(\hat{R} \hat{f}) \cong T(\hat{R} \hat{e})$.

$(\Leftarrow)$ If $R$ is a direct sum of primary rings then every faithful projective $R$-module must be a generator and every factor ring of $R$ has the same property. According to [9, Th. 3.3] or [11, Th. 1.10] a quasi-projective $R$-module $M$ is faithful and projective over a factor ring of $R$. Thus $M$ is a generator over that factor ring and has the double centralizer property by [5, Th. 1]. This completes the proof.

Camillo [2] calls a ring $R$ balanced in case each of its right modules has the double centralizer property (equivalently, each factor ring of $R$ is $Q F-1$ in the sense of Thrall [18]). He proved that every balanced ring is semi-perfect (for properties of semi-perfect and perfect rings, see [1]) with nil radical $N$ and observed that a direct sum of rings is balanced if and only if so is each of the direct summands. In the case where $N$ is nilpotent, our theorem together with a recent theorem of Morita and Tachikawa reduces the study of balanced rings to that of local rings. ( $R$ is local if $R / N$ is a division ring.) 
COROLlaRY 1. Every indecomposable semi-primary balanced ring is Morita equivalent to a local balanced ring.

Proof. According to Theorem 2 of the appendix of [14], if $R$ and $S$ are Morita equivalent rings (i. e., if their categories of modules are isomorphic in the sense of Morita [13]) then $R$ is $Q F-1$ when $S$ is. Moreover, from the results of [13] it follows that if $R$ and $S$ are Morita equivalent then each factor ring of $R$ bears the same relationship to a factor ring of $S$. Thus $Q F-1$ and balanced are both categorical concepts. But every primary ring $R$ is Morita equivalent to a local ring (in fact, isomorphic to a full matrix ring over a local ring $e R e, e$ a primitive idempotent in $R$ ); and according to our theorem indecomposable balanced semi-primary rings are primary.

A ring $R$ has dominant dimension, dom. dim. $(R)$, at least $n$ in case there is an exact sequence

$$
0 \rightarrow R \rightarrow E_{1} \rightarrow \cdots \rightarrow E_{n}
$$

with $E_{i}$ an injective projective left $R$-module, $i=1, \cdots, n$ (see, for example, [16]). A left artinian ring is $Q F-3$ in case it has dominant dimension at least 1 . Thus, according to [7, Th. 3.6], a left artinian ring is (right artinian and) generalized uniserial if and only if each of its factor rings has dominant dimension at least 1 . Every $Q F$ ring has infinite dominant dimension (Nakayama conjectured the converse, at least for finite dimensional algebras (see [15])) and, according to the proof of Lemma 2 in Nakayama's [17], a ring is uniserial [12] (= a direct sum of primary generalized uniserial rings) if and only if each of its factor rings is $Q F$. Now, because every faithful projective (equivalently, every faithful injective) module over a $Q F-3$ ring $R$ has the double centralizer property precisely when dom.dim. $(R) \geqq 2$ (see [8] and [16]), we see that this condition on the factor rings is much stronger than necessary.

COROLlaRY 2. A left artinian ring $R$ is uniserial if (and only if) each factor ring of $R$ has dominant dimension at least 2.

REMARKS. (a) The sufficiency part of the theorem is valid for right perfect rings. The necessity part holds for any semi-perfect ring in which central idempotents can be lifted modulo $N^{2}$, as they can in a semi-primary ring.

(b) It is not difficult to prove that a left module over a right perfect ring is rationally complete (i. e., has no proper rational extension (see [6, p. 58])) if and only if its only essential extensions by simple modules are by simple submodules of itself. Thus the argu- 
ment of the theorem shows that a semi-primary ring $R$ is a direct sum of primary rings if and only if the left regular representation of each factor ring of $R$ is rationally complete (cf., [3]).

(c) A left artinian ring is a direct sum of primary rings if and only if each of its left quasi-injective modules has the double centralizer property. This follows from [8, Th. 5], [9, Corollary 1.3], and our present theorem.

(d) Since every factor ring of a uniserial ring is $Q F$, uniserial rings are balanced. In [2] Camillo proved that balanced commutative rings are uniserial. By Corollary 2, balanced generalized uniserial rings are also uniserial. In fact, all the balanced rings that we know of are uniserial rings. ${ }^{1}$

I wish to thank Anne Koehler for communicating to me her observation that quasi-projective modules over a perfect commutative ring have the double centralizer property. This led me to the corresponding implication of the present theorem.

\section{REFERENCES}

1. H. Bass, Finitistic dimension and a homological generalization of semi-primary rings, Trans. Amer. Math. Soc. 95 (1960), 466-488.

2. V. P. Camillo, Balanced rings and a problem of Thrall, Trans. Amer. Math. Soc. (to appear)

3. R. Courter, Finite direct sums of complete matrix rings over perfect completely primary rings, Canad. J. Math. 21 (1969), 430-446.

4. B. Eckmann and A. Schopf, Über injective Modulen, Arch. Math. 4 (1953), 75-78.

5. C. Faith, A general Wedderburn theorem, Bull. Amer. Math. Soc. 73 (1967), 65-67.

6. L Lectures on injective modules and quotient rings, Springer-Verlag, Berlin-Heidelberg-New York, 1967.

7. K. R. Fuller, On indecomposable injectives over artinian rings, Pacific J. Math. 29 (1969), 115-135.

8. - Double centralizers of injectives and projectives over artinian rings Illinois J. Math. (to appear).

9. - On direct representations of quasi-injectives and quasi-projectives, Arch. Math. 20 (1969), 495-502.

10. R. E. Johnson and E. T. Wong, Quasi-injective modules and irreducible rings, J. London Math. Soc. 36 (1961), 260-268.

11. A. Koehler, Quasi-projective and quasi-injective modules, Pacific J. Math. (to appear)

12. G. Köthe, Verallgemeinerte Abelsche Gruppe mit hyperkomplexem Operatorring, Math. Zeit. 39 (1934), 31-44.

13. K. Morita, Duality of modules and its applications to the theory of rings with

1 As this goes to press we note that J. P. Jans has formally conjectured that balanced artinian rings are indeed uniserial. Moreover, he has proved that this is the case for algebras over algebraically closed fields. 
minimum condition, Sci. Rep. Tokyo Kyoiku Daigaku 6 (1958), 85-142.

14. K. Morita and $H$. Tachikawa, $Q F-3$ rings unpublished

15. B. J. Mueller, The classification of algebras by dominant dimension, Canad. J. Math, 20 (1968), 398-409.

16. Dominant dimension of semi-primary rings, J. Reine Angew. Math. 232 (1968), 173-179.

17. T. Nakayama, Note on uniserial and generalized uniserial rings, Proc. Imp. Acad. Japan 16 (1940), 285-289.

18. R. M. Thrall, Some generalizations of quasi-Frobenius algebras, Trans. Amer. Math. Soc. 64 (1948), 173-183.

19. L. E. T. Wu and J. P. Jans, On quasi-projectives, Illinois J. Math. 11 (1967), 439-448.

Recived November 7, 1969.

The UNIVERSITY OF IOWA 



\title{
PACIFIC JOURNAL OF MATHEMATICS
}

\author{
EDITORS
}

\author{
H. SAMELSON \\ Stanford University \\ Stanford, California 94305 \\ RichaRd PIERCE \\ University of Washington \\ Seattle, Washington 98105
}

J. DugundJI

Department of Mathematics

University of Southern California

Los Angeles, California 9.0007

RICHARD ARENS

University of California

Los Angeles, California 9.0024

\section{ASSOCIATE EDITORS}

E. F. BeCKenBACH
B. H. NeumanN

F. WoLE

K. YoSHIDA

\section{SUPPORTING INSTITUTIONS}

\author{
UNIVERSITY OF BRITISH COLUMBIA \\ CALIFORNIA INSTITUTE OF TECHNOLOGY \\ UNIVERSITY OF CALIFORNIA \\ MONTANA STATE UNIVERSITY \\ UNIVERSITY OF NEVADA \\ NEW MEXICO STATE UNIVERSITY \\ OREGON STATE UNIVERSITY \\ UNIVERSITY OF OREGON \\ OSAKA UNIVERSITY \\ UNIVERSITY OF SOUTHERN CALIFORNIA
}

\author{
STANFORD UNIVERSITY \\ UNIVERSITY OF TOKYO \\ UNIVERSITY OF UTAH \\ WASHINGTON STATE UNIVERSITY \\ UNIVERSITY OF WASHINGTON \\ AMERICAN MATHEMATICAL SOCIETY \\ CHEVRON RESEARCH CORPORATION \\ TRW SYSTEMS \\ NAVAL WEAPONS CENTER
}

The Supporting Institutions listed above contribute to the cost of publication of this Journal, but they are not owners or publishers and have no responsibility for its content or policies.

Mathematical papers intended for publication in the Pacific Journal of Mathematics should be in typed form or offset-reproduced, (not dittoed), double spaced with large margins. Underline Greek letters in red, German in green, and script in blue. The first paragraph or two must be capable of being used separately as a synopsis of the entire paper. The editorial "we" must not be used in the synopsis, and items of the bibliography should not be cited there unless absolutely necessary, in which case they must be identified by author and Journal, rather than by item number. Manuscripts, in duplicate if possible, may be sent to any one of the four editors. Please classify according to the scheme of Math. Rev. Index to Vol. 39. All other communications to the editors should be addressed to the managing editor, Richard Arens, University of California, Los Angeles, California, 90024.

50 reprints are provided free for each article; additional copies may be obtained at cost in multiples of 50 .

The Pacific Journal of Mathematics is published monthly. Effective with Volume 16 the price per volume (3 numbers) is $\$ 8.00$; single issues, $\$ 3.00$. Special price for current issues to individual faculty members of supporting institutions and to individual members of the American Mathematical Society: $\$ 4.00$ per volume; single issues $\$ 1.50$. Back numbers are available.

Subscriptions, orders for back numbers, and changes of address should be sent to Pacific Journal of Mathematics, 103 Highland Boulevard, Berkeley, California, 94708.

PUBLISHED BY PACIFIC JOURNAL OF MATHEMATICS, A NON-PROFIT CORPORATION

Printed at Kokusai Bunken Insatsusha (International Academic Printing Co., Ltd.), 7-17, Fujimi 2-chome, Chiyoda-ku, Tokyo, Japan. 


\section{Pacific Journal of Mathematics}

\section{Vol. 34, No. $2 \quad$ June, 1970}

Shair Ahmad, On the oscillation of solutions of a class of linear fourth order

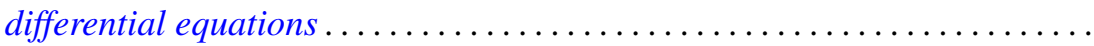

Leonard Asimow and Alan John Ellis, Facial decomposition of linearly

compact simplexes and separation of functions on cones ..............

Kirby Alan Baker and Albert Robert Stralka, Compact, distributive lattices of

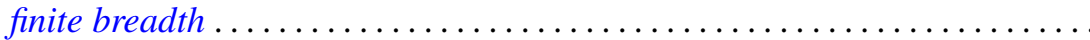

James W. Cannon, Sets which can be missed by side approximations to

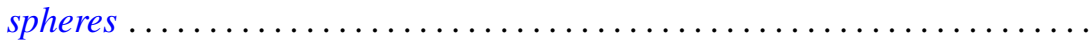

Prem Chandra, Absolute summability by Riesz means .................. 335

Francis T. Christoph, Free topological semigroups and embedding topological semigroups in topological groups....

Henry Bruce Cohen and Francis E. Sullivan, Projecting onto cycles in smooth, reflexive Banach spaces.................................

John Dauns, Power series semigroup rings .......................

Robert E. Dressler, A density which counts multiplicity ................

Kent Ralph Fuller, Primary rings and double centralizers ................

Gary Allen Gislason, On the existence question for a family of products.......

Alan Stuart Gleit, On the structure topology of simplex spaces .............

William R. Gordon and Marvin David Marcus, An analysis of equality in

certain matrix inequalities. $I \ldots \ldots \ldots \ldots \ldots \ldots \ldots \ldots$

Gerald William Johnson and David Lee Skoug, Operator-valued Feynman

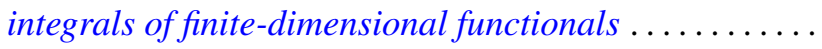

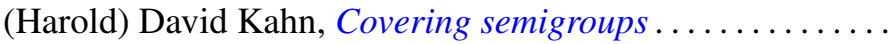

Keith Milo Kendig, Fibrations of analytic varieties

Norman Yeomans Luther, Weak denseness of nonatomic measures on perfect,

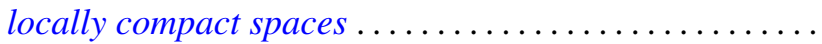

Guillermo Owen, The four-person constant-sum games; Discriminatory solutions on the main diagonal ...

Stephen Parrott, Unitary dilations for commuting contractions

Roy Martin Rakestraw, Extremal elements of the convex cone $A_{n}$ of

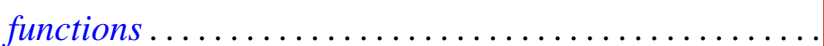

Peter Lewis Renz, Intersection representations of graphs by

William Henry Ruckle, Representation and series summability of complete

biorthogonal sequences.

F. Dennis Sentilles, The strict topology on bounded sets ...

Saharon Shelah, A note on Hanf numbers ...

Harold Simmons, The solution of a decision problem for several classes of rings. . .

Kenneth S. Williams, Finite transformation formulae involving the Legendre 